Check for updates

Cite this: RSC Adv., 2018, 8, 16503

Received 8th March 2018

Accepted 19th April 2018

DOI: $10.1039 / c 8 r a 02072 h$

rsc.li/rsc-advances

\section{Double-layered microsphere based dual growth factor delivery system for guided bone regeneration}

\author{
Chun Xu, $\mathbb{D} \dagger^{\mathrm{a}}$ Jia Xu, $\dagger^{\mathrm{b}}$ Lan Xiao, $\dagger^{\mathrm{ac}}$ Zhihao Li, ${ }^{\mathrm{a}}$ Yin Xiao, ${ }^{c}$ Matthew Dargusch, ${ }^{\mathrm{d}}$ \\ Chang Lei, ${ }^{\text {e }}$ Yan He (iD *a and Qingsong Ye (iD *a
}

\begin{abstract}
Microsphere based drug delivery systems show great advantages for tissue engineering. However, it is still a big challenge to fabricate microspheres with capability in delivering and controlled releasing multiple growth factors. In the present study, double-layered microspheres consisting of an inner-layer of small core particles and an outer-layer of big shell particles were developed to sequentially release cell homing factors (SDF-1) and osteoinductive growth factors (BMP-2) for bone regeneration. In vitro release testing showed that bioactivity of both growth factors retained within the microspheres and differential release of SDF-1 and BMP-2 was achieved. Microspheres with both growth factors showed an obvious chemotaxis effect on preosteoblasts by inducing more cell migration. In osteoinductive ability tests, the microspheres with both growth factors showed higher ALP activity and more mineralized modules than control groups after culturing for 2 weeks. The expression of bone development transcription factors (Runx2, OCN, Osterix) as well as Smad signals (Smad 1, 5, 8) showed higher gene expression in the dual growth factor group. Our results suggest that a double-layered microsphere system enhances the recruitment of osteogenic cells and osteoinduction, which provides a promising platform for bone regeneration.
\end{abstract}

\section{Introduction}

Microsphere based drug delivery systems receive great interest for various biomedical applications where therapeutic agents can be released in a controllable manner. ${ }^{1}$ Polymeric microspheres are ideal vehicles for many controlled delivery applications due to their good biocompatibility, high bioactivity and sustained drug release characteristics. ${ }^{2-4}$ Compared to other drug delivery systems, microspheres offer advantages including the ability to encapsulate and protect therapeutic molecules with large sizes such as growth factors as well as easy administration through needles etc. ${ }^{5-7}$ Various techniques such as spray-drying, ${ }^{8}$ emulsion, ${ }^{9}$ phase separation ${ }^{\mathbf{1 0}}$ and electrospray ${ }^{\mathbf{1 1}}$ have been developed to prepare microspheres. Conventional solid microspheres usually release the loaded drugs using

${ }^{a}$ School of Dentistry, The University of Queensland, Brisbane, Queensland, 4006, Australia.E-mail:h.he@uq.edu.au; a.ye@uq.edu.au

${ }^{b}$ College of Medicine and Dentistry, James Cook University, Cairns 4878, Australia ${ }^{c}$ Institute of Health and Biomedical Innovation, Queensland University of Technology, Kelvin Grove Campus, Brisbane, 4006, Australia

${ }^{d}$ ARC Research Hub for Advanced Manufacturing of Medical Devices, Centre for Advanced Materials Processing and Manufacturing, School of Mechanical and Mining Engineering, The University of Queensland, Brisbane, 4006, Australia

${ }^{e}$ Australian Institute for Bioengineering and Nanotechnology, The University of Queensland, Brisbane, QLD, 4072, Australia

$\dagger$ Those authors contributed equally to this work. a single release behavior due to their simple structure. For biomedical applications, there is an increased requirement for the development of multi-drug delivery methods which can release the drugs in a controlled and sequential way. For example for bone tissue engineering it is demonstrated that controlled release of different biochemical cues (e.g. growth factors) in a designed manner recruits more host circulating stem cells and fastens the bone regeneration process, which may provide systematic therapeutic effects compared to a single cue. ${ }^{12-15}$

One important application of microsphere based delivery systems is for the treatment of large bone defects caused by infections, injuries or congenital deformity, which remains a clinical challenge. Bone is a highly dynamic tissue that bone resorption and osteoblastic bone formation. Under physiological conditions, bone repair can be performed by attracting mesenchymal stem cells (MSCs) to the defect sites and further directing their differentiation towards osteoblasts under a precise regulation of various growth factors at different time points. For example, stromal cell derived factor-1 (SDF-1), a member of the CXC chemokine family, induces the migration of progenitor/stem cells to the bone defect area during the acute phase and initiates the regeneration process. ${ }^{12,16}$ Bone morphogenetic protein-2 (BMP-2), is an important osteoinductive growth factor, has been approved by the US Food and Drug Administration (FDA) for clinical use for the induction of bone involves continuous remodeling accompanied by osteoclastic 
formation via the stimulation of osteogenic differentiation of MSC. ${ }^{17}$ More importantly, it has been reported that SDF-1 signaling contributes to the osteogenic differentiation of mesenchymal cells induced by bone morphogenetic protein-2 (BMP-2). ${ }^{12}$ A delivery system that sequentially releases designed growth factors, for example first to release chemokine which triggers the migration of stem cells and progenitor cells to injury sites and secondly to release osteoinductive growth factors which are necessary for the following osteogenic differentiation, may be of great benefit for the treatment of bone defects, which is difficult to achieve by conventional microspheres with simple structure.

To address such challenges, microspheres with core-shell structure have been developed to load multi-drugs and to tail the release profile. For example, a double-emulsion approach has been used to prepare microcapsules using immiscible solutions. ${ }^{7}$ However, important limitations exist with these approaches such as uncontrolled architecture of the synthesized microcapsules, and the use of organic solvents in the synthetic process denaturing the fragile biochemical cues e.g. growth factors. It is still a challenge to fabricate a microsphere based delivery system that is able to sequentially release growth factors while maintaining their bioactivities and functionalities.

Electrospray provides a simple and robust technique for fabricating microsphere with different sizes from a broad range of biocompatible polymers. ${ }^{\mathbf{1 1}}$ Alginate, has been widely used in pharmaceutical applications due to its excellent biocompatibility, minimal binding interactions with tissue cells and can be ionically crosslinked into hydrogels by divalent cations, e.g. calcium. ${ }^{18}$ Alginate degrades slowly due to dissociation of the ionic crosslinks, where drug release is achieved. The drug release rate can be also controlled via modification of alginate with other polymers such as chitosan. ${ }^{19}$ Alginate microspheres can be easily obtained with electrospray method. ${ }^{20}$ With a twostep electrospray process, alginate microspheres with doublelayered structure can be obtained with small core microspheres and large shell microspheres. Such architectures can encapsulate different types of drugs and control the release kinetics which are suitable for growth factors delivery for bone repair and may provide extra benefits.

In this study, a novel drug-delivery system based on doublelayered microspheres was fabricated, which differentially released SDF-1 and BMP-2. The initially released SDF-1 facilitates the recruitment of MSCs to the injury sites, subsequently followed by the release of BMP-2 inducing the osteogenic differentiation of MSCs. The drug release profiles were tested in vitro. The homing effect of stem cells/progenitors into the microspheres through a chemotaxis assay with a trans-wells system. The osteoinductive ability of the synthesized MDS with SDF-1/BMP-2 were tested on bone marrow stromal stem cells (BMSCs).

\section{Materials and methods}

\subsection{Materials}

Alginic acid sodium salt (AS, from brown algae, bioreagent), chitosan (molecular weight 50-190 kDa, 75-85\% deacetylated), albumin-fluorescein isothiocyanate conjugate (FITC-albumin, $\geq 7$ mol FITC per mol albumin), albumintetramethylrhodamine isothiocyanate bovine (TRITCalbumin, $\geq 0.5 \mathrm{~mol}$ TRITC per mol albumin), calcium chloride (anhydrous, bioreagent), Tween 80 (premium) and acetic acid $(\geq 99.7 \%)$ were purchased from Sigma-Aldrich Corp. Stromalcell derived factor-1 (SDF-1) and bone morphogenetic protein2 (BMP-2) were purchased from GenScript, United States. All chemicals were used as received without further purification.

\subsection{Preparation of alginate double-layered microsphere}

Double-layered microspheres were prepared via a two steps electrospray method. A stem cell homing inducer (SDF-1) was loaded into the shell layer and a bone tissue regeneration inducer (BMP-2) was loaded into the core layer. In the first step, BMP-2 solution (containing 0.1\% Tween 80, 1.5\% AS and 6.67 $\mu \mathrm{g} \mathrm{mL}{ }^{-1}$ BMP-2) was introduced into a home-made electrospray device. $^{20}$ The solution was loaded in a syringe that was controlled by an electrical pump ejecting the core layer via a blunt stainless steel needle, which served as a charged spinneret. A grounding dish filled with $4 \% \mathrm{CaCl}_{2}$ aqueous solution was placed underneath the needle at a distance of $10 \mathrm{~cm}$. A high voltage $(30 \mathrm{kV})$ was applied between the needle and grounding dish by a power supply (TianJin Dongwen High Voltage Power Supply Co., Ltd, China). Under this high voltage, AS/BMP-2 microdroplets were jetted toward the $\mathrm{CaCl}_{2}$ solution and microspheres were formed. Those AS/BMP-2 microspheres were collected by centrifugation (1000 rpm, $5 \mathrm{~min}$ ) and washed with distilled water. Then the microspheres were coated with a layer of chitosan by stirring in $0.1 \%$ chitosan/acetic acid solution (1\%, $\mathrm{pH}$ was adjusted to 5.5 ) for 30 minutes. The products were washed with acetic acid once and distilled water twice.

In the second step, those AS/BMP-2 microspheres coated with chitosan were mixed with AS/SDF-1 solution containing $0.1 \%$ Tween $80,1.5 \%$ AS and $3.34 \mu \mathrm{g} \mathrm{mL}{ }^{-1}$ SDF-1 by gentle stirring. Then the mixture was introduced to the electrospray device and went through a second electrospray process with a voltage of $30 \mathrm{kV}$. The products were washed with distilled water three times and freeze-dried for storage. Those microspheres containing both SDF- 1 and BMP-2 were denoted as S-B group. For comparison, microspheres with BMP-2 only (in the core layer, B group), SDF-1 only (in the shell layer, S group) or without any growth factor (O group) were also fabricated.

For the fabrication of alginate microspheres with 3 chitosan layers, first alginate microspheres were prepared using above method. Then those microspheres were dispersed in $0.1 \%$ chitosan/acetic acid solution (1\%, pH was adjusted to 5.5) for 30 minutes. After washing with water, the obtained microspheres were stirred in $0.1 \%$ alginate solution for $30 \mathrm{~min}$. This process repeated for three times until the microspheres were coated with three layers of chitosan (chitosan-alginate-chitosan-alginate-chitosan). After that those microspheres went through another electrospun process to form the double-layered structure. For lysozyme loaded microspheres $4 \mathrm{mg} \mathrm{mL}^{-1}$ lysozyme water solution was added to the alginate $(1.5 \%)$ and Tween 80 $(0.1 \%)$ solution to fabricate the inner microsphere. 
In order to confirm the location of loaded growth factors inside the microspheres, FITC-albumin and TRITC-albumin were used as model proteins and loaded into this doublelayered microsphere using the same procedure. The samples were observed under a fluorescence microscope (LEICA DMi8, Leica Microsystems, Germany).

\subsection{Characterization of the microspheres}

The morphology of the double-layered microspheres was observed with an optical microscope (LEICA DMi8, Leica Microsystems, Germany) and scanning electron microscopy (Phenom G2 Pro, Netherland). For optical microscopy observation, the microparticles were dispersed in phosphate buffered saline (PBS) and placed on a glass slide. Zeta potential measurements were carried out at $25^{\circ} \mathrm{C}$ using a Zetasizer NanoZS from Malvern instruments. The samples were dispersed at $10 \mathrm{mM} \mathrm{NaCl}(\mathrm{pH} 7.4)$ solution. For SEM analysis, the microparticles were dehydrated by a series of ethanol $(70 \%, 80 \%, 90 \%$ and $100 \%$ ) and acetone buffer. Then the samples were placed on an SEM mount with carbon tape, air-dried, coated with platinum. The SEM images were obtained using Phenom G2 pro (Phenom-world, the Netherlands) with an accelerating voltage of $5 \mathrm{kV}$.

\subsection{Determination of protein release}

The release of lysozyme from double-layered microspheres in vitro was quantified by Qubit Protein Assay Kit (Thermo Fisher Scientific, Australia). The microspheres ( $2 \mathrm{mg})$ were incubated in $5 \mathrm{~mL}$ DMEM with vigorous shaking at $37{ }^{\circ} \mathrm{C}$. At the desired time points $(0,1,2,3,4,6,8 \mathrm{~h})$ the solution was centrifuged and $1 \mathrm{~mL}$ supernatant was collected. The solution was topped up with addition of $1 \mathrm{~mL}$ fresh DMEM. The concentrations of lysozyme in the supernatant were tested using Qubit Protein Assay Kit with Qubit 2 Fluorometer (Invitrogen). The release tests were repeated three times.

The release of SDF-1 and BMP-2 from double-layered microspheres in vitro was quantified via an enzyme-linked immunosorbent assay (ELISA) method. The microspheres $(\sim 2$ $\mathrm{mg}$ ) were incubated in $5 \mathrm{~mL}$ DMEM with vigorous shaking at $37{ }^{\circ} \mathrm{C}$. At the desired time points $(0,1,2,3,4,6,8,24 \mathrm{~h})$ the solution was centrifuged and $1 \mathrm{~mL}$ supernatant was collected and stored at $-20{ }^{\circ} \mathrm{C}$. The solution was topped up with addition of $1 \mathrm{~mL}$ fresh DMEM. The concentrations of SDF-1 and BMP-2 were tested using SDF-1 and BMP-2 ELISA kit (Life Tech Australia Pty Ltd, Australia) according to the manufacturer's instructions. Cumulative release ratio was calculated as the ratio of the cumulative mass of SDF-1 or BMP-2 released at each time interval to their initial input amount in the microspheres, respectively. The release tests were repeated three times.

\subsection{Cell culture}

BMSCs were obtained from 8-10 weeks old C57 mice (mBMSCs) as described in a previous study. ${ }^{21}$ All experiment procedures were performed in accordance with Australian code for the care and use of animals for scientific purposes (Australian Government, National Health and Medical Research Council,
Australia, 2013) and guidelines to promote the wellbeing of animals used for scientific purposes (Australian Government, 2008) and were approved by the Animal Ethics Committee of the Queensland University of Technology (Brisbane, Australia). Briefly, femurs were harvested after the mouse was sacrificed. The femurs were washed three times by PBS with $1 \%(\mathrm{v} / \mathrm{v})$ penicillin/streptomycin (P/S; Gibco®, Life Technologies Pty Ltd., Australia). Both ends of femurs were removed, and then the bone marrow was flushed with Dulbecco's modified Eagle's medium (DMEM; Gibco®, Life Technologies Pty Ltd., Australia) containing $1 \% \mathrm{P} / \mathrm{S}$ into a $50 \mathrm{~mL}$ tube. BMSCs in bone marrow were collected by centrifugation $\left(1000 \mathrm{rpm}, 5 \mathrm{~min}, 20{ }^{\circ} \mathrm{C}\right)$, washed once with PBS (containing 1\% P/S) and then resuspended in DMEM containing $10 \%(\mathrm{v} / \mathrm{v})$ fetal bovine serum (FBS; In Vitro Technologies, Australia) and 1\% P/S. The cellular suspension was transferred into a culture flask and incubated with $5 \% \mathrm{CO}_{2}$ at $37{ }^{\circ} \mathrm{C}$. By day 3, the non-adherent cells were removed. Adherent cells were then continually incubated in DMEM with $10 \%$ FBS and $1 \% \mathrm{P} / \mathrm{S}$. Culture medium was changed every 2-3 days. When reaching $90 \%$ confluence, the cells were passaged by 2 min exposure to $0.25 \%$ trypsin (containing $1 \mathrm{mM}$ EDTA, Gibco®, Life Technologies Pty Ltd., Australia). Cells within 5 passages were used for in vitro osteogenesis. Pre-osteoblast cell line MC3T3-E1 was obtained from American Type Culture Collection (ATCC) and cultured in DMEM containing $10 \%(\mathrm{v} / \mathrm{v}) \mathrm{FBS}$; and $1 \% \mathrm{P} / \mathrm{S}$.

\subsection{MTT (3-(4,5-dimethylthiazol-2-yl)-2,5- diphenyltetrazolium bromide) assay}

To evaluate the effect of microspheres on the cellular viability of mBMSCs, MTT assay was performed as previously described. ${ }^{22}$ Briefly, mBMSCs (3000 cells per well, three wells per group) were stimulated by cultured medium with graded concentrations $(0$, $50,100,250 \mathrm{mg} \mathrm{mL}^{-1}$ ) of microspheres in $\mathrm{O}, \mathrm{S}, \mathrm{B}$, and $\mathrm{S}-\mathrm{B}$ groups, respectively. After 24 h, 72 h, 7 d's culture, the culture medium was replaced by $100 \mu \mathrm{L}$ of fresh culture medium, and $10 \mu \mathrm{L}$ MTT solution $\left(0.5 \mathrm{mg} \mathrm{mL}^{-1}\right.$, Sigma-Aldrich Pty Ltd., Sydney, Australia) was added into each well. Cells were incubated with MTT for $4 \mathrm{~h}$, and then the medium was removed; 200 $\mu \mathrm{L}$ of dimethyl sulfoxide was added into each well to solubilize the formazan product, which was then read at $495 \mathrm{~nm}$ by a microplate reader (SpectraMax, Plus 384, Molecular Devices, Inc., United States) to measure the absorbance. All the results were normalized against the absorbance of blank wells. All the measurements were repeated three times.

\subsection{Chemotaxis assay}

A pre-osteoblast cell line MC3T3-E1 was used to test the homing effect of the microspheres using a trans-well system (Becton Dickinson Labware, Franklin Lakes, NJ, USA). Three groups were established: blank control group (C group), $\mathrm{S}$ group (microspheres with $3.3 \mu \mathrm{g} \mathrm{mL}^{-1}$ SDF-1 in the shell layer), S-B group (microspheres with $3.3 \mu \mathrm{g} \mathrm{mL}{ }^{-1}$ SDF-1 in the shell and $6.6 \mu \mathrm{g} \mathrm{mL}{ }^{-1}$ BMP-2 in the core). The microspheres with $600 \mu \mathrm{L}$ complete culture medium were placed in the bottom layer. The cells $(100 \mu \mathrm{L}$ cell suspension, 36000 cells per $\mathrm{mL})$ were seeded 
in the companion culture inserts ( $8 \mu \mathrm{m}$ pore size). After culture for 24,72 and $120 \mathrm{~h}$, cells in the basolateral chamber were stained with $0.1 \%$ crystal violet solution. To quantify the cell migration, the blue sediments were dissolved with $33 \%$ acetic acid and the absorbance was measured by a microplate reader (Infinite ${ }^{\circledR} 200$ PRO, Tecan Trading AG, Switzerland) under $595 \mathrm{~nm}$.

\subsection{In vitro osteogenesis of mBMSCs}

To induce the in vitro osteogenesis, mBMSCs were seeded in 6well plates at a density of $2 \times 10^{5}$ cells per well. After 24 h postseeding, culture medium was replaced by the osteogenic medium (DMEM containing 10\% FBS, 1\% P/S, $10 \mathrm{mM} \beta$-glycerophosphate, $50 \mu \mathrm{M}$ ascorbic acid and $100 \mathrm{nM}$ dexamethasone; Sigma-Aldrich Pty Ltd., Sydney, Australia), which was changed every three days. To test the effect of S-B group on the mBMSCs osteogenesis, S-B microspheres at a dose of $100 \mathrm{mg}$ $\mathrm{mL}^{-1}$ was added into the osteogenic medium; the cells applied with $\mathrm{O}, \mathrm{S}$ and $\mathrm{B}$ microspheres served as the controls; cells without microsphere application served as blank controls (group C). Cells with three days' stimulation were harvested for RNA extraction and ALP activity assay. Cells stimulated for 14 days were harvested for Alizarin Red S staining.

\subsection{RNA extraction, cDNA synthesis, and real time quantitative-PCR (RT-qPCR)}

Total RNA of mBMSCs was extracted by the TRIzol reagent (Ambion®, Life Technologies Pty Ltd., Australia). The cDNA was then synthesized from $1 \mu \mathrm{g}$ total RNA by SensiFAST ${ }^{\mathrm{TM}}$ cDNA Synthesis Kit (Bioline Pty Ltd., Australia) following the manufacturer's instruction. RT-qPCR was performed through the QuantStudio $^{\text {TM }} 7$ Flex Real-Time PCR System (Applied Biosystems, Australia) with SYBR ${ }^{\circledR}$ Green reagent (Applied Biosystems, Australia) according to the manufacturer's instructions. The RT-qPCR primers used in this study were listed in Table 1 , the designs of which were based on cDNA sequences from the National Centre for Biotechnology Information (NCBI) sequence database. The tests were conducted on the following target genes: $i B S P, C O L-1$, Osterix, OCN, OPN, RUNX2, Smad 1/5/8. The house keeping gene GAPDH and $\beta$ - actin were used as control. All reactions were run in triplicate for three independent experiments. Relative gene expression was normalized against GAPDH or $\beta$-actin and calculated as previously described. ${ }^{23}$

\subsection{ALP activity assay}

The cells with three days osteogenesis induction were harvested for ALP activity assay by ALP assay buffer (Abcam, Cambridge, UK). Protein concentration was measured by the BCA Protein Assay Kit (Thermo Fisher Scientific, VIC, Australia). The ALP activity was tested by Alkaline Phosphatase Assay kit (Colorimetric) (Abcam, Cambridge, UK) following the manufacture's instruction. All tests were performed in triplicate for three independent experiments. All the results were expressed as the calculated ALP activity/protein concentrations.

\subsection{Alizarin Red $S$ staining}

After 14 days of osteogenic induction, cells were fixed with $4 \%$ PFA for $20 \mathrm{~min}$ at room temperature, and then rinsed with distilled water. After that, the cells were stained with 1\% Alizarin Red S (Sigma-Aldrich Pty Ltd., Sydney, Australia) solution (pH: 4.1-4.3) for $20 \mathrm{~min}$ at room temperature. After washing three times with distilled water, the samples were air-dried and observed under microscope (Eclipse TS100, Nikon Australia Pty Ltd.). The quantification of Alizarin Red staining was performed by analyzing the percentage of positive area through the Image J software. The average number of five randomly chosen areas of each sample was calculated. Samples from three independent replications were analyzed for Alizarin Red S staining intensity. All measurements were performed in a double-blind manner by two independent observers.

\subsection{Statistical analysis}

All statistical analysis was carried out using GraphPad Prism 7 (GraphPad Software, Inc., USA). Statistical analysis was performed using the Student's unpaired $t$-test for two groups' comparisons and through one-way variance analysis (ANOVA) for multiple comparisons $(p \leq 0.05)$.

Table 1 Sequence of primers used for RT-PCR test

\begin{tabular}{|c|c|c|}
\hline Gene & Forward sequences & Reverse sequences \\
\hline iBSP & 5'CAG AGG AGG CAA GCG TCA CT3' & 5'CTG TCT GGG TGC CAA CAC TG3' \\
\hline COL-1 & 5’AGA ACA GCG TGG CCT ACA TG3' & 5'TCC GGT GTG ACT CGT GC3' \\
\hline Osterix & 5'AGC GAC CAC TTG AGC AAA CAT3' & 5'GCG GCT GAT TGG CTT CTT CT3' \\
\hline OCN & 5'ACC TAG CAG ACA CCA TGA GGA C3' & $5^{\prime}$ GGG GAC TGA GGC TCC AAG3' \\
\hline OPN & 5'CAA TGA AAG CCA TGA CCA CAT GG3' & $5^{\prime}$ CTC ATC TGT GGC ATC AGG ATA CTG3' \\
\hline RUNX2 & 5'CAGGCAGGTGCTTCAGAACT3' & 5'ACTCTGGCTTTGGGAAGAGC3' \\
\hline Smad 1 & 5'CGAGCCGGCGCTAAC3' & 5'CCCTGTTTCCACCCAAGGAG3' \\
\hline Smad 5 & 5'GGGAGATAAGGATCCGCGCT3' & 5'GCTTCTTCTTAGTGCAAGTCACAA3' \\
\hline Smad 8 & 5'TGAGTATCACTCTCTTGAGTAAGC3' & 5'CACGGGGAAAGAGGATGAGAC3’ \\
\hline GAPDH & 5'TCAGCAATGCCTCCTGCAC3' & 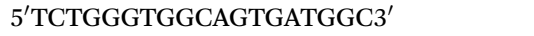 \\
\hline$\beta$-actin & 5'CTGTCGAGTCGCGTCCACC3' & 5'ATTCCCACCATCACACCCTGG3' \\
\hline
\end{tabular}




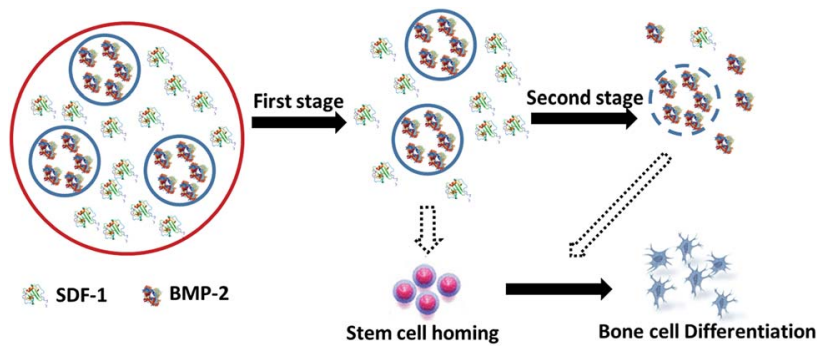

Fig. 1 Schematic illustration of the double-layered microspheres based growth factors delivery system.

\section{Results}

\subsection{Characterization of the double-layered microspheres}

As shown in Fig. 2A, double-layered microspheres were welldispersed particles with a uniform spherical shape with the size of around $380 \mu \mathrm{m}$. The double-layered structure could be clearly observed in Fig. 2B where several small particles (core particles) were embedded in a big particle (shell particle). To confirm that two different growth factors were loaded into the different parts of double-layered microspheres, fluorescence dye labelled proteins (albumin) was used as the drug model and added during the formation of core particles and shell particles, respectively. From the fluorescence microscopy image (Fig. 2C) red fluorescence was observed from the core particles, which were incorporated inside large microspheres with green fluorescence, confirming the loading of two proteins in corresponding layers.

\subsection{SDF-1 and BMP-2 release profiles from the double- layered microspheres}

The release profiles of SDF-1 and BMP-2 from double-layered microspheres loaded with SDF-1 and BMP-2 were investigated in vitro. The amount of released growth factors were tested by corresponding ELISA kits. As shown in Fig. 2D, an initial burst release of SDF-1 from microspheres was noticed in the first $6 \mathrm{~h}$, with about $80 \%$ of loaded SDF- 1 being set free to the medium. BMP-2 showed delayed and mild release profile comparing to SDF-1. 50\% of BMP-2 was release in around 4 hours while $50 \%$ of SDF-1 was released in $2 \mathrm{~h}$. The release rate can be further tuned by changing the coating layers of chitosan. To demonstrate this, we coated the alginate microspheres with three layers of chitosan (chitosan-alginate-chitosan-alginate-chitosan, Fig. 3A), and the $\zeta$ potential changes (minus with alginate and positive after chitosan, Fig. 3B) indicated the successful coating of each layer. After that those microspheres went through another electrospun process to form the doublelayered structure. We used lysozyme as a protein model and loaded it into the inner microspheres. Microscopy image (Fig. 3B) showed those microspheres with 3 layers of chitosan had similar structure with those microspheres with 1 layer of chitosan and lysozyme release profile (Fig. 3C) showed a slower release rate. $50 \%$ of lysozyme was release at around 6.5 hours in the case of microspheres with 3 chitosan layers while it took only $3.5 \mathrm{~h}$ for those with 1 chitosan layer.

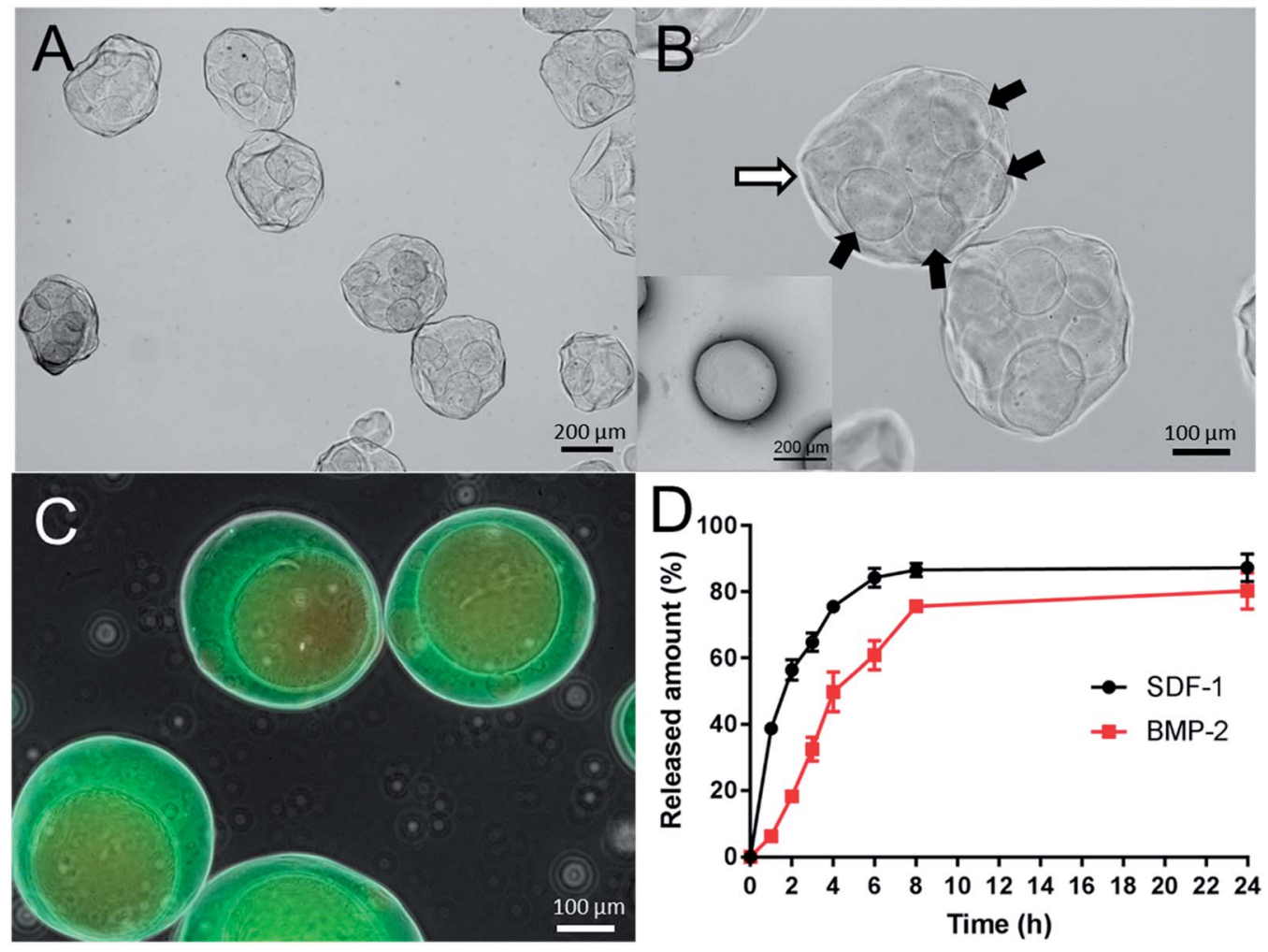

Fig. 2 Optical microscope images of the double-layered microspheres (A and B) and fluorescence microscope image after loaded with florescent dyes (C). Inset in (B) showed the SEM image of double-layered microsphere. (D) showed the release profiles of SDF-1 and BMP-2 from growth factor loaded double-layered microspheres. 

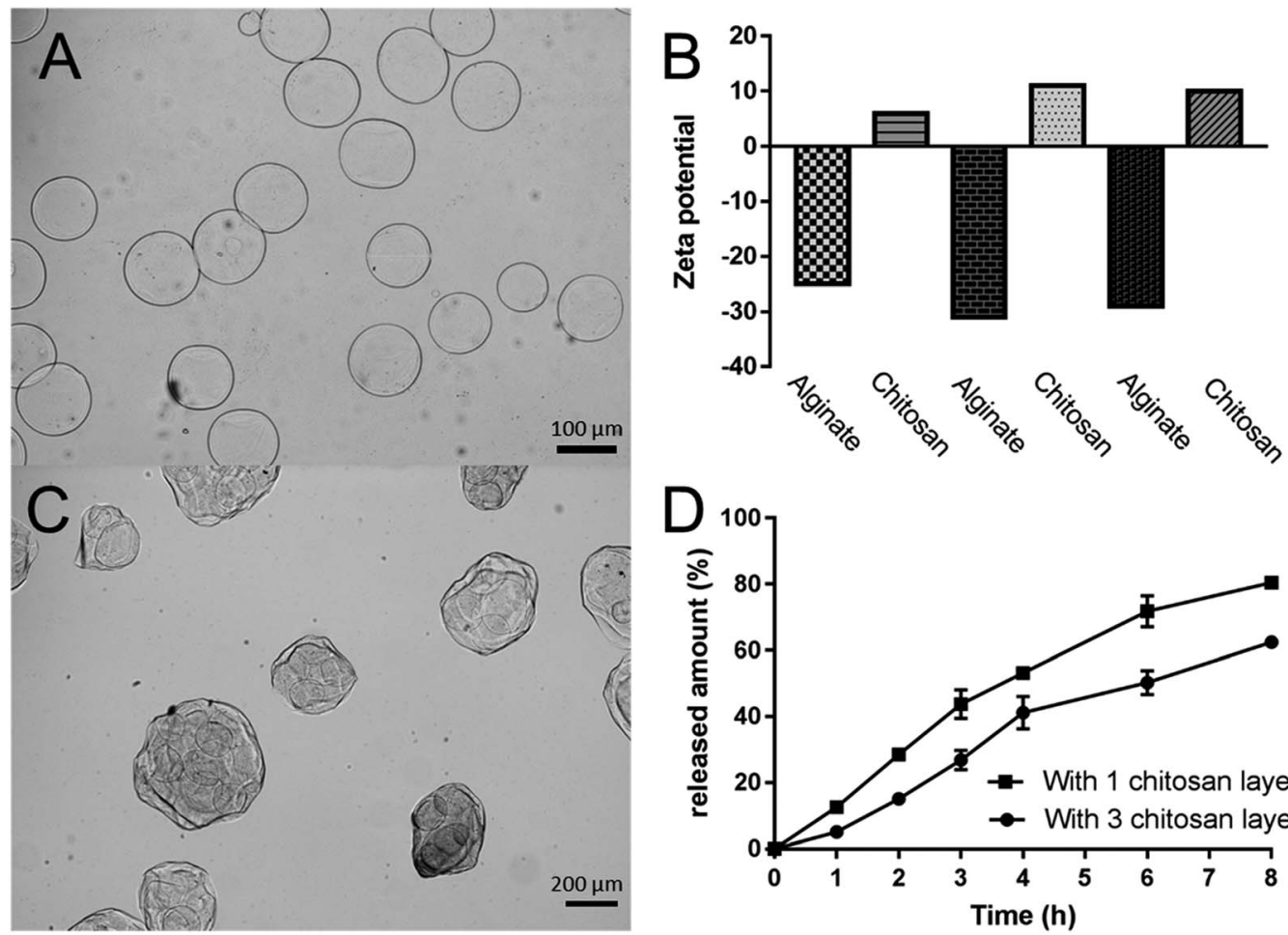

Fig. 3 Optical microscope image of the inner alginate microspheres with three layers of chitosan (A) and the $\zeta$ potential change during the layerby-layer coating process (B). (C) showed the microscope image of double-layered microspheres with three layers of chitosan and (D) showed the release profiles of lysozyme from double-layered microspheres with one and three layers of chitosan.

\subsection{Effect of the double-layered microspheres on cell viability of mBMSCs}

MTT assay was performed to consecutively evaluate the effects of the double-layered microspheres with multiple cytokines (SDF-1/BMP-2) on the proliferation of mBMSCs. For O group, no significant difference regarding mBMSC-proliferation could be identified among dose groups $\left(0 / 50 / 100 / 250 \mathrm{mg} \mathrm{mL}^{-1}\right)$ at each time point ( $24 \mathrm{~h}, 72 \mathrm{~h}, 7 \mathrm{~d}$ ), except for $250 \mathrm{mg} \mathrm{mL}^{-1}$ on day 7 (Fig. 4A). For the S group (Fig. 4B), S-Ms at doses of 50 and $100 \mathrm{mg} \mathrm{mL}^{-1}$ leaded to significantly increased proliferation of mBMSCs at $72 \mathrm{~h}$-culture; besides that, no significant difference
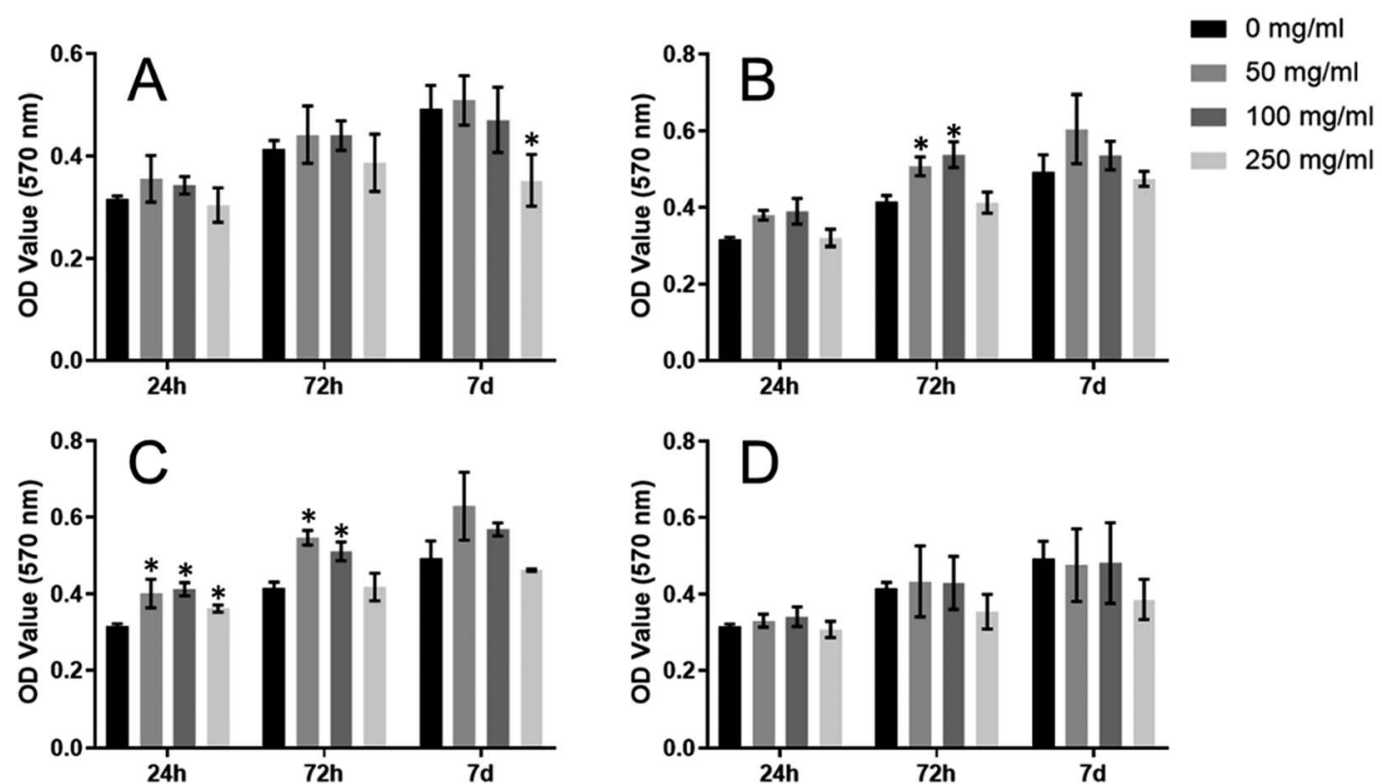

Fig. 4 Effects on cell proliferation of growth factor loaded double layer microspheres ((A) O, no growth factors were loaded in microspheres; (B) S, SDF-1 was loaded in microspheres; (C) B, BMP-2 was loaded in microspheres; (D) S-B, SDF-1 and BMP-2 were loaded in microspheres). 
could be found among dose groups at each time point. As shown in Fig. 4C, B-Ms application did not result in any reduction in the proliferation of mBMSCs; whereas B-Ms at 50/ $100 / 250 \mathrm{mg} \mathrm{mL}^{-1}$ leaded to a significant increase in cellular growth at $24 \mathrm{~h}$. Also, B-Ms at $50 / 100 \mathrm{mg} \mathrm{mL}^{-1}$ induced cellular growth at 72 h. For S-B group (Fig. 4D), no significant difference could be found within dose groups at each time point. Compared with the blank-control $\left(0 \mathrm{mg} \mathrm{mL}^{-1}\right)$, the $\mathrm{O}, \mathrm{S}$, B andS$B$ groups at the doses of 50 and $100 \mathrm{mg} \mathrm{mL}^{-1}$ did not inhibit the proliferation mBMSCs. Therefore, $100 \mathrm{mg} \mathrm{mL}^{-1}$ was applied in the following study.

\subsection{Double-layered microspheres with SDF-1 induced the cell migration}

To test the effect of SDF-1-loading microspheres on cellular migration, a chemotaxis assay was performed by the trans-well system. As shown in Fig. 5, in comparison with the blank control group (C group, Fig. 5A and B), S-Ms application

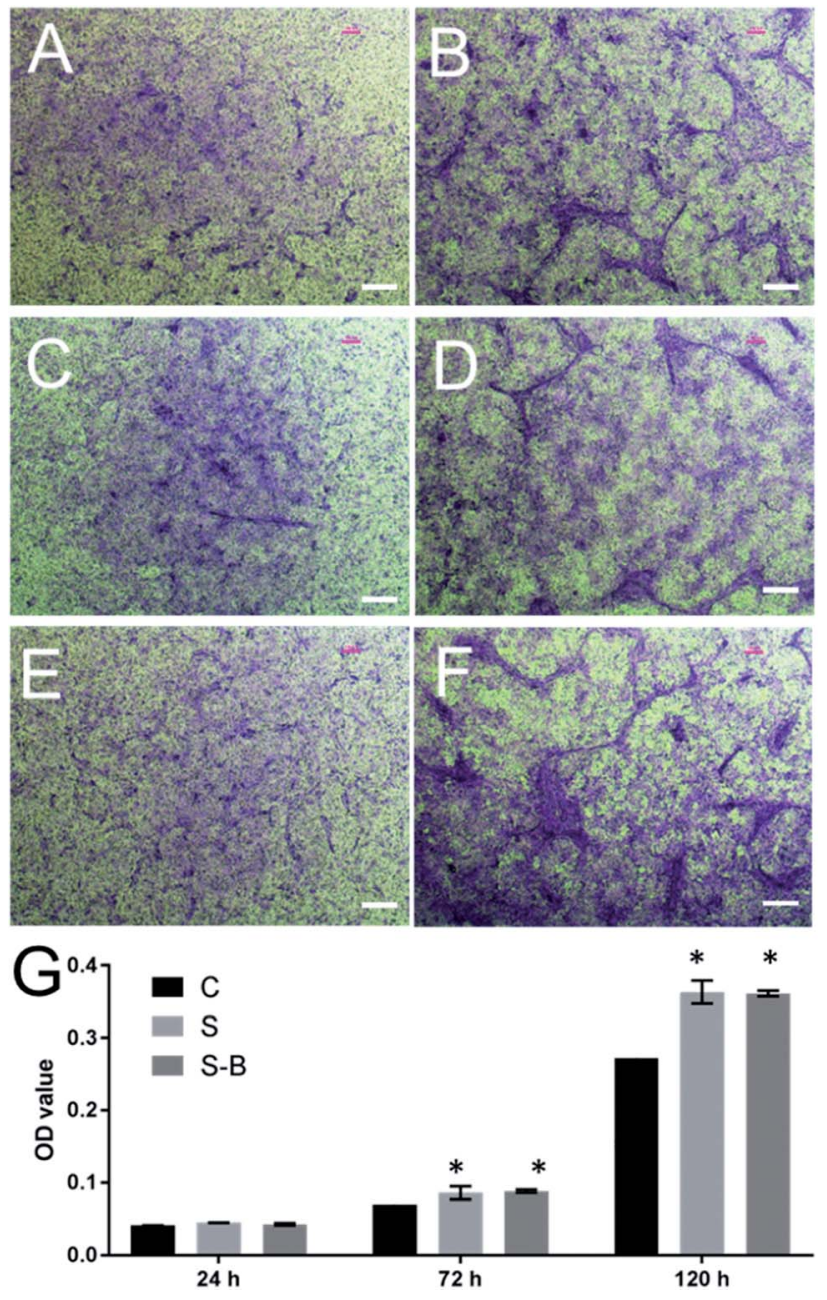

Fig. 5 Optical microscope images of MC3T3-E1 cells in chemotaxis assay after treated with control group ( $A$ and $B), S$ group $(C$ and $D)$ and S-B group ( $E$ and F) for $72 \mathrm{~h}$ ( $\mathrm{A}, \mathrm{C}$ and $\mathrm{E}$ ) and $120 \mathrm{~h}$ (B, D and F). (G) shows the optical density (OD) of control group and experimental groups in chemotaxis assay. *Significantly different from control group $(p<0.05)$. Scale bar $=200 \mu \mathrm{m}$. attracted more cells migrating from the upper insert to the lower chamber (S group, Fig. 5C and D). Accordingly, the OD values of the $\mathrm{S}$ group were significantly higher than those of the control group at $72 \mathrm{~h}$ and $120 \mathrm{~h}$, which further proved that cellular migration was significantly induced by S-Ms.

\subsection{SDF-1/BMP-2 double-delivery microspheres significantly induced the osteogenic differentiation of mBMSCs}

As shown in Fig. 6, in comparison with the blank control group (C group), mBMSCs applied with B and S-B microsphere groups showed significantly induced mRNA levels ( 2 to 4 folds) of the osteogenic markers: iBSP, COL-1, Osterix, OPN, and RUNX2. Compared with the $\mathrm{C}$ group, cells with $\mathrm{O}$ and $\mathrm{S}$ microsphere application did not show significantly induced expression regarding iBSP, COL-1, Osterix, and RUNX2; while significantly increased mRNA levels regarding these factors were found in $\mathrm{B}$ and S-B groups in comparison with $\mathrm{O} / \mathrm{S}$ groups. As shown in Fig. $6 \mathrm{D}$ and $\mathrm{E}$, in comparison with the $\mathrm{C}$ group, all the groups showed induced expression of OCN and OPN; whereas the S-B group did not show any significant difference on OCN expression when compared with $\mathrm{O}$ and $\mathrm{S}$ groups. The mRNA levels of Smad 1/5/8 were significantly induced in mBMSCs within S-B group. Moreover, the cells in S-B group significantly upregulated ALP activity, as compared with group $\mathrm{C}, \mathrm{O}$ and $\mathrm{S}$ (Fig. 7).

\subsection{SDF-1/BMP-2 double-delivery microspheres significantly induced mineralization of mBMSCs}

To test the effect of the S-B group on mineralization, Alizarin Red $S$ staining was performed to identify the mineralization nodules. As shown in Fig. 8D and E, both B and S-B microsphere applications showed enhanced mineralization of mBMSCs, as compared with the controls (group C, O and S) (Fig. 8A-C). The positive areas of Alizarin Red $\mathrm{S}$ were calculated; as shown in Fig. 8F. Positive areas in both B and S-B groups significantly increased, as compared with those in the blank control (C group) and the $\mathrm{O} / \mathrm{S}$ groups (Fig. 8F). No significant difference could be found within the control groups (group C, O and S) or between the $\mathrm{B}$ and the S-B groups.

\section{Discussion}

Microsphere based drug delivery systems have been one of the most popular research topics in recent decades. This is due to the potential benefits of achieving the long-time effects of the drug while minimizing the side-effects, therefore making great improvements to the current drug-based clinical therapies. ${ }^{\mathbf{2 4 - 2 6}}$ Solid microspheres usually designed for single drug loading and release, have been unable to meet the requirements of combination therapies in bone regeneration, in which multiple drugs of diverse therapeutic purposes/effects are used to improve the outcomes of treatments. ${ }^{27-30}$ Core-shell microspheres offer several advantages over solid spheres such as the ability to load multiple drugs and precisely control the release order, timing and dose. ${ }^{31}$ Another big challenge for drug delivery systems is to maintain the bioactivity of loaded drugs 

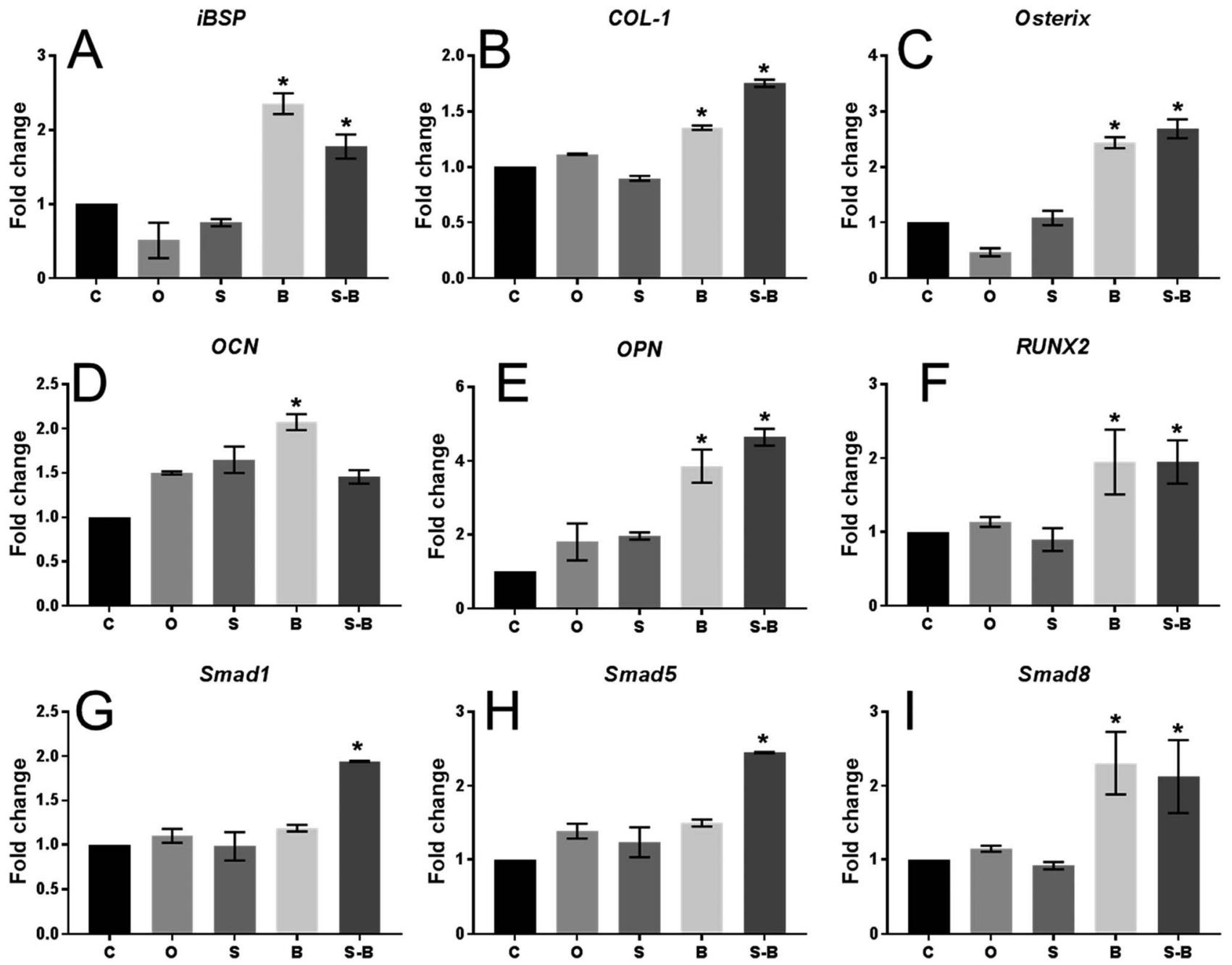

Fig. 6 Real-time PCR analysis of osteogenic genes (iBSP, COL-1, Osterix, OCN, OPN, RUNX2) and Smad signaling genes (Smad 1, 5, 8) under osteogenic induction for 7 days $(n=3$ per group; $* p<0.05)$.

especially for those protein drugs. ${ }^{32-35}$ In this study, the novel alginate core-shell microspheres were prepared by electrospray to achieve a differential delivery of growth factors and also maintain the biological functions. As shown in Fig. 1, stromal cell derived factor-1 (SDF-1), a member of the CXC chemokine family, was loaded into the shell part to trigger the migration of MSCs to injury sites during the acute phase of bone repair and encourage participation in regeneration. Bone morphogenetic protein-2 (BMP-2), the key osteoinductive growth factor, which has been approved by the US FDA for clinical use to induce bone formation via the enhancement of osteogenic differentiation, was loaded into the core part. With our rational design, the effects of the sequential and sustained release of SDF-1 and BMP-2 on the migratory and osteogenic capacity of MSCs and bone regeneration were assessed in vitro.

From the characterization data (Fig. 2B), it can be observed that the double-layered microsphere consisted of several small particles (inner-layer core particles) and one big shell (outerlayer particle). To test the multiple drug delivery capacity of this microsphere, red/green fluorescence dye labelled albumins were loaded into the inner core and outer shell. As shown in
Fig. 2C, the red core was embedded into the large green shell, indicating that this double-layer microsphere was successful fabricated and suitable for double growth factor (SDF-1 and BMP-2) loading.

To test the release properties of the double-layer microsphere, ELISA assay was performed. As shown in Fig. 2D, SDF-1 and BMP-2 were differentially released from the microspheres;

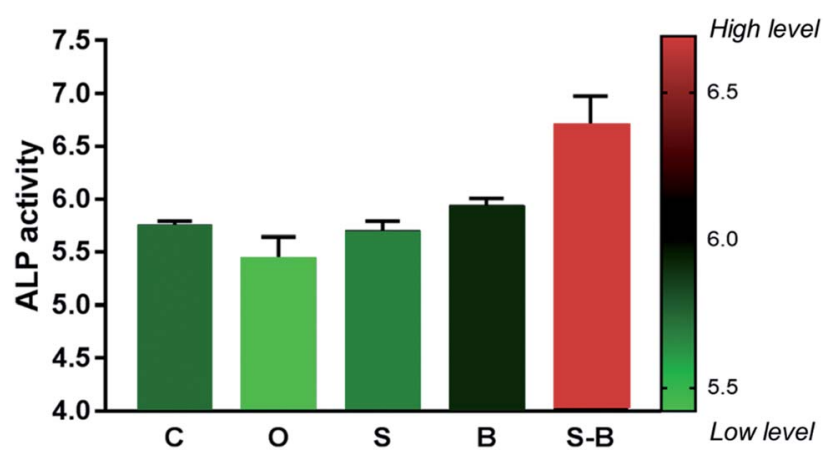

Fig. 7 Quantitative ALP activity of BMSCs cultured in different treatment groups at day 7 ( $n=3$ in each group; $\left.{ }^{*} p<0.05\right)$. 


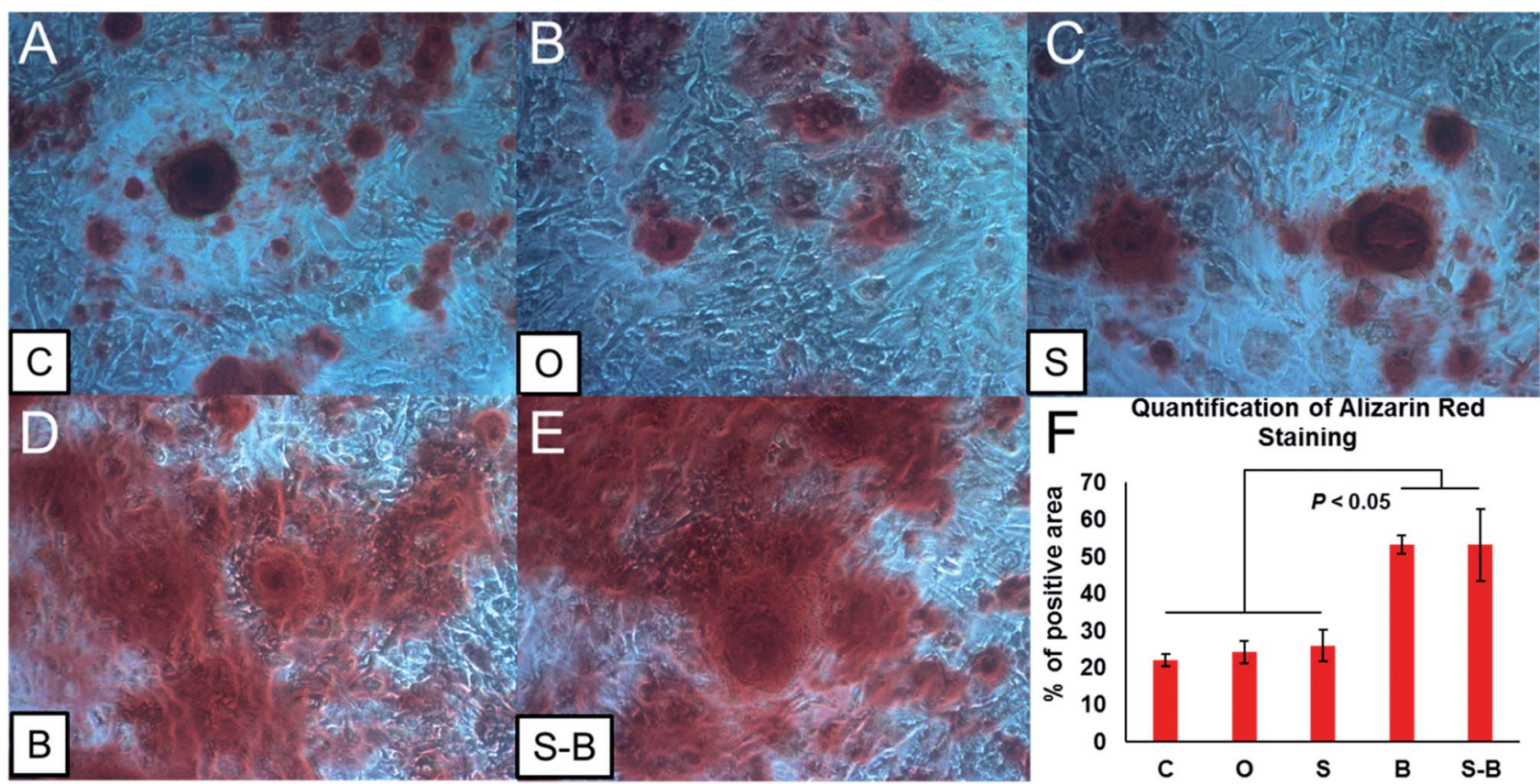

Fig. 8 Alizarin Red staining of BMSCs (A-E) with different treatment group sunder osteogenic induction for 14 days. F showed the quantification of Alizarin Red staining.

SDF-1 release was faster than that of BMP-2. Hence, it can be proposed that the SDF-1/BMP-2 double-delivery microspheres could release SDF-1 firstly to induce MSCs recruitment, and then release BMP-2 to facilitate their osteogenic differentiation. The biocompatibility of this microsphere was then tested by MTT assay, and from the results in Fig. 4, it could be observed that the double-layer microsphere merely affected the viability of mBMSCs after 7 days' stimulation, indicating the good biocompatibility of this microsphere.

The cell chemotaxis properties of SDF-1 and SDF-1/BMP-2 loading microspheres were then verified. As shown in Fig. 5, microspheres containing SDF-1 and SDF-1/BMP-2 showed significantly induced cell migration after $72 \mathrm{~h}$, indicating that the released SDF-1 could effectively attract more MSCs to migrate towards the injury sites in vivo.

The effect of the SDF-1/BMP-2 double-delivery microspheres on osteogenesis was then systematically examined in vitro. The current study found that SDF-1/BMP-2 double-delivery microspheres showed superior osteoinductive capacity, as the S-B-Ms application induced the osteogenic differentiation of mBMSCs. As shown in Fig. 6, in comparison with the controls that carried no microspheres, void microspheres and microspheres with SDF-1, mBMSCs with S-B-Ms showed significantly enhanced mRNA levels in the number of osteogenic markers. Similarly, mBMSCs with B-Ms also showed increased expression of osteogenic markers, indicating that the BMP-2 released by microspheres could effectively induce the osteogenic differentiation. The BMP-2 mediated osteogenesis has been shown to occur because of the Smad signaling, ${ }^{36-38}$ therefore, the mRNA levels of Smad 1/5/8 were also examined; and the cells with S-B-Ms showed significantly induced expression of Smad 1/5/8 (Fig. 6G-I). This further proved that the effect of S-B-Ms- mediated osteoinduction was due to the released BMP-2. Moreover, cells applied with S-B-Ms showed the highest activity of ALP (Fig. 6) the key factor in osteogenic differentiation. ${ }^{39,40}$ Therefore, the osteoinductive properties of the SDF-1/ BMP-2 double-delivery microspheres were confirmed. It was observed that there were differences between the B and S-B groups on the expression of osteogenic markers, such as iBSP, Col-1, OCN, and Smad 1/5. This might be due to the different BMP-2 release profiles between the B-Ms and S-B-Ms. The effect of the SDF-1/BMP-2 double-delivery microspheres on mineralization was then tested. It was observed that after 14 days osteoinduction, cells applied with S-B-Ms and B-Ms achieved much more mineralization than the blank/O-/S-controls (Fig. 7). All these results demonstrated the osteoinductive ability of SDF1/BMP-2 double-delivery microspheres.

When all results in this study are considered together, a novel double-drug delivery system has been successfully prepared, which showed good capability in loading and release of multiple drugs. The double-layer structure not only guarantees the loading of different drugs, but also makes the system able to release the drugs in a differential manner. The SDF-1/ BMP-2 double-delivery microspheres showed superior performance in inducing the migration and osteogenic differentiation of MSCs in vitro, which was due to the differentially released SDF-1 and BMP-2. Therefore, the current study provides a promising novel approach for drug delivery and bone tissue regeneration. Further in vivo studies should be performed to further this effect in vivo.

\section{Conclusion}

Double layered microspheres with dual growth factors delivery ability were successfully fabricated via two steps electrospray 
method. Cell homing test showed an obvious chemotaxis effect on preosteoblasts of our double layered microspheres. For osteoinductive ability tests, S-B MC showed much higher ALP activity and more mineralized modules than control groups after 2 weeks' culture. The RT-PCR tests of key transcription factors (Runx2, OCN, Osterix) that regulate bone development and important bone forming signals (Smad 1, 5, 8) showed much higher gene expression in dual growth factors group. These results suggest the potential of our microspheres as drug delivery system for biomedical applications.

\section{Conflicts of interest}

There are no conflicts to declare.

\section{Acknowledgements}

This work was supported by the Australian Research Council DE120101666 and NSFC 81701032. CX would like to thank the support of National Health \& Medical Research Council of Australia (NHMRC) Early Career Fellowship. YH would like to appreciate the support of Women's Academic Fund, Advance Queensland (WAF-4425969-189). MSD would like to acknowledge the support of the ARC Research Hub for Advanced Manufacturing of Medical Devices.

\section{References}

1 N. K. Varde and D. W. Pack, Expert Opin. Biol. Ther., 2004, 4, 35-51.

2 H. Zhang, S. Y. Tong, X. Z. Zhang, S. X. Cheng, R. X. Zhuo and H. Li, J. Phys. Chem. C, 2007, 111, 12681-12685.

3 W. R. Gombotz and S. F. Wee, Adv. Drug Delivery Rev., 2012, 64, 194-205.

4 J. Panyam and V. Labhasetwar, Adv. Drug Delivery Rev., 2012, 64, 61-71.

5 G. Yang, Z. Q. Li, H. Zhao, M. Zhang and D. H. Sun, J. Biomater. Tissue Eng., 2017, 7, 708-714.

6 Z. H. Hu, F. Wu, W. Yuan, M. He and T. Jin, J. Controlled Release, 2017, 259, E190-E191.

7 K. K. Kim and D. W. Pack, BioMEMS and Biomedical Nanotechnology, Springer, Boston, USA, 2006.

8 Y. Huh, H. J. Cho, I. S. Yoon, M. K. Choi, J. S. Kim, E. Oh, S. J. Chung, C. K. Shim and D. D. Kim, Eur. J. Pharm. Sci., 2010, 40, 9-15.

9 Q. C. Zhang, K. Tan, Z. Y. Ye, Y. Zhang, W. S. Tan and M. D. Lang, Mater. Sci. Eng., C, 2012, 32, 2589-2595.

10 H. Ghanbar, C. J. Luo, P. Bakhshi, R. Day and M. Edirisinghe, Mater. Sci. Eng., C, 2013, 33, 2488-2498.

11 Y. Fukui, T. Maruyama, Y. Iwamatsu, A. Fujii, T. Tanaka, Y. Ohmukai and H. Matsuyama, Colloids Surf., A, 2010, 370, 28-34.

12 X. F. Shen, Y. X. Zhang, Y. Gu, Y. Xu, Y. Liu, B. Li and L. Chen, Biomaterials, 2016, 106, 205-216.

13 J. Ratanavaraporn, H. Furuya, H. Kohara and Y. Tabata, Biomaterials, 2011, 32, 2797-2811.
14 M. Yamamoto, A. Hokugo, Y. Takahashi, T. Nakano, M. Hiraoka and Y. Tabata, Biomaterials, 2015, 56, 18-25.

15 Osteoporosis Australia Medical \& Scientific Advisory Committee, Osteoporosis Australia, http:// www.osteoporosis.org.au/statistics-burden-disease.

16 W. Ji, F. Yang, J. L. Ma, M. J. Bouma, O. C. Boerman, Z. Chen, J. J. J. P. van den Beucken and J. A. Jansen, Biomaterials, 2013, 34, 735-745.

17 J. A. Sterling and S. A. Guelcher, Curr. Osteoporos. Rep., 2014, 12, 48-54.

18 L. B. Priddy, O. Chaudhuri, H. Y. Stevens, L. Krishnan, B. A. Uhrig, N. J. Willett and R. E. Guldberg, Acta Biomater., 2014, 10, 4390-4399.

19 X. F. Niu, P. Chen, X. L. Jia, L. Z. Wang, P. Li, L. Yang, Y. L. Wang and Y. B. Fan, Int. J. Pharm., 2014, 469, 214-221.

20 J. Xu, Y. He, J. F. Ma and Q. S. Ye, Mater. Lett., 2017, 188, 152155.

21 P. Tropel, D. Noel, N. Platet, P. Legrand, A.-L. Benabid and F. Berger, Exp. Cell Res., 2004, 295, 395-406.

22 C. Wu, Y. Zhou, W. Fan, P. Han, J. Chang, J. Yuen, M. Zhang and Y. Xiao, Biomaterials, 2012, 33, 2076-2085.

23 A. L. Bookout and D. J. Mangelsdorf, Nucl. Recept. Signaling, 2003, 1, e012.

24 E. Soussan, S. Cassel, M. Blanzat and I. Rico-Lattes, Angew. Chem., Int. Ed. Engl., 2009, 48, 274-288.

25 V. Torchilin, Eur. J. Pharm. Biopharm., 2009, 71, 431-444.

26 S. Ganta, H. Devalapally, A. Shahiwala and M. Amiji, J. Controlled Release, 2008, 126, 187-204.

27 Y. Kakeji, E. Oki, A. Egashira, N. Sadanaga, I. Takahashi, M. Morita, Y. Emi and Y. Maehara, Oncology, 2009, 77, 49-52.

28 O. F. Bathe, S. Ernst, F. R. Sutherland, E. Dixon, C. Butts, D. Bigam, D. Holland, G. A. Porter, J. Koppel and S. Dowden, BMC Cancer, 2009, 9, 156.

29 H. B. Fung, E. A. Stone and F. J. Piacenti, Clin. Ther., 2002, 24, 1515-1548.

30 B. A. Luxon, M. Grace, D. Brassard and R. Bordens, Clin. Ther., 2002, 24, 1363-1383.

31 T. Okuda, K. Tominaga and S. Kidoaki, J. Controlled Release, 2010, 143, 258-264.

32 S. Narisawa, M. Nagata, C. Danyoshi, H. Yoshino, K. Murata, Y. Hirakawa and K. Noda, Pharm. Res., 1994, 11, 111-116.

33 R. Ishino, H. Yoshino, Y. Hirakawa and K. Noda, Chem. Pharm. Bull., 1992, 40, 3036-3041.

34 I. R. Wilding, S. S. Davis, M. Bakhshaee, H. N. Stevens, R. A. Sparrow and J. Brennan, Pharm. Res., 1992, 9, 654-657.

35 S. Ueda, T. Hata, S. Asakura, H. Yamaguchi, M. Kotani and Y. Ueda, J. Drug Targeting, 1994, 2, 35-44.

36 B. S. Kim, H.-J. Kang, J.-Y. Park and J. Lee, Exp. Mol. Med., 2015, 47, e128.

37 P. C. Bessa, M. Casal and R. Reis, J. Tissue Eng. Regener. Med., 2008, 2, 1-13.

38 A. B. Celil, J. O. Hollinger and P. G. Campbell, J. Cell. Biochem., 2005, 95, 518-528.

39 M. Fallon, M. Whyte and S. Teitelbaum, Lab. Invest., 1980, 43, 489-494.

40 E. E. Golub and K. Boesze-Battaglia, Curr. Opin. Orthop., 2007, 18, 444-448. 